deamination of all amino acids in tissues containing no universal amino-oxhydrases. Ever since the first publications on trans-amination, $I$ have pointed out the possible role of trans-amination in the synthesis of aminoacids in vegetable organisms, where the aminodicarboxylic acids apparently belong to the first organic products of nitrogen assimilation ${ }^{8}$. Recently, Virtanen ${ }^{3}$ and Euler, Adler et al. ${ }^{4}$ have reported experimental evidence supporting, though not conclusively proving, the role of the trans-amination mechanism in amino acid synthesis.

We wish to point out that the properties of l-deaminase (Krebs, Bernheim) indicate the complex nature of this enzyme and suggest that oxidative deamination of the $l$-amino acids is also probably due to the co-operation of aminopherases, glutamic or aspartic dehydrogenase and catalytic amounts of the respective dicarboxylic acids. Experimental verification of this hypothesis is in progress. If such be the case, it would follow that the dicarboxylic amino- and $\alpha$-ketoacids function as intermediate carriers not only in the interconversion of amino acids by trans-amination, but also in their synthesis from ammonia and deamination, thus forming a transition channel between mineral and organic nitrogen.

Summary.-The results of recent investigations concerning the transfer of amino-groups between amino- and ketoacids are discussed. These investigations led to the discovery of a new group of intracellular enzymes, termed aminopherases. A survey is given of the range of specificity and mode of action of these enzymes. The biological significance of the aminopherases and of the dicarboxylic amino- and ketoacids is discussed with a view to their probable role as key substances in the biological formation and breakdown of amino acids.

${ }^{1}$ Braunstein, A. E., Kritzmann, M. G., Enzymologia, 2, 129 (1937). Biochimia (Moscow) , 2, 242, 860 (1937) ; 3, 590, 693 (1938); 4 , No. 2 (1939).

${ }^{2}$ Kritzmann, M., Enzymologia, 5, 44 (1938). Biochimia, 3, 28 (1938).

${ }^{3}$ Virtanen and Laine, NATURE, 141, 748 (1938).

${ }^{4}$ Euler, H., Adler, E., et al., Z. physiol. Chem., 2ృ4, 61 ; 255, 14, 27.

${ }^{5}$ Braunstein, A. E., (unpublished data).

${ }^{8}$ Bychkov, S. M., Biochimia (1939, in the Press).

Kritzmann, M. G., C.R. (Doklady) Acad. Sci. U.R.S.S., 21, 42 (1938). NATURE (this issue, $p, 603$ ).

\& Biochimia, 2, 257 (1937).

\title{
The Atmosphere as a Raw Material*
}

$I^{\mathrm{N}}$ the middle of the seventeenth century, the phlogiston theory was generally accepted. Scientific study of the atmosphere only began in the last quarter of that century, and within fifty years of Stahl's formulation of the theory, it had been displaced, and oxygen had been recognized, isolated and identified. In little more than a century, the composition of air was known to 1 per cent, and not very long afterwards, Ramsay completed the story with his discovery of the inert constituents. At the present time, gases which are only present to the extent of one drop in 130 gallons are articles of everyday commerce.

Although use is now being made of the atmosphere as a raw material of industry, our demands are as yet quite unlikely to affect the stock appreciably. England and Scotland have twenty or more factories taking the oxygen from 20 million cubic feet daily, but this large amount ceases to seem dangerous when we remember that the population of those countries uses 600 million cubic feet daily in the ordinary eourse of respiration; animals add their demands to this figure, and the total is again vastly increased by the supply required for the daily combustion of wood, coal and petrol. The case is in marked contrast to that of coal. Mankind is continuously oxidizing carbon, and yet rapidly diminishing the amount of the only agent-vegetation--that can renew his exhalations to a useful atmosphere. The energy from sunlight received in a growing season by an acre of land is equivalent to that obtained by the combustion of 243 tons of anthracite. If 25 bushels of corn are reaped from the acre, they return energy equivalent to only 0.3 tons of anthracite. Nor is the prospect from water power very favourable. It is estimated that the hydro-electric power available

* Substance of the presidential address delivered to Section B (Chemistry) of the Australian and New Zealand Association for the Advancement of Science, by Russell Grimwade, C.B.E., on January 12, 1939. from the whole rainfall of America is about equal to the present power production from coal.

So far as the production of carbon dioxide by human beings is concerned, it is interesting to calculate that a man exhales about $3 \mathrm{lb}$. per day; a eucalyptus tree of the mountain ash type, 200 feet high and weighing 24 tons, is capable of breaking down about one third of this amount and returning the oxygen to the atmosphere. It is clear therefore that the tendency to replace vegetation of this type by buildings, or even by cereal crops, carries with it a potential danger to the oxygen supply.

It was about the year 1885 that man began to abstract oxygen from the air for its own sake, when the barium oxide process was first used commercially. At first, much of the oxygen was used for therapy, and for the limelight. Now that this is replaced by electric light, about 98 per cent of the oxygen prepared is used in engineering, either for metallurgical, fabricating, or cutting purposes. Of course, the oxygen is no longer extracted by chemical processes, but by liquefaction and subsequent rectification, in plants that can make 4,000 gallons an hour for days on end. The fractional distillation of liquid air depends for its success on the heat exchange tray devised by Kuhni, and known as a 'foam plate'. In it, a disk is built up in a true spiral, with about 10,000 holes to the square inch.

Turning now to the inert gases, argon is present to the extent of about 1 per cent, but was only detected in 1894 by Ramsay and Rayleigh. It is now separated directly in a state of comparative purity from commercial oxygen distilling apparatus. At the present time, about 10 million cubic feet are used annually to fill the 1,000 million electric lamps produced in the same time. Neon exists only to the extent of 1 part in 55,000 , but its characteristic spectrum has led to the use of thousands of litres annually in 'neon lights' so widely used in advertising signs. 
Helium, present to the extent of one part in 245,000 , or 50 tons in the atmosphere above each square mile of the earth's surface, is the lightest uninflammable gas, and is thus of especial use in lighterthan-air craft. For this purpose it is collected from the gases of certain bore-holes in the United States, where it is present to the extent of 1-2 per cent. It is of particular value medically in that an artificial atmosphere of helium and oxygen can prevent Caisson disease or 'the bends', a malady, due to the solubility of nitrogen in human blood serum, to which divers and others working under increased pressure are subject. Krypton, occurring only to the extent of 1 part in a million, and xenon, only one tenth as abundant, are not used very extensively as yet, but the fact that they increase the efficiency of an electric lamp filament by 10 and 20 per cent respectively, suggests that they will eventually be in common use.

The remaining constituent of the atmosphere, nitrogen, constitutes 81 per cent of its volume. F1om about 1830, nitrogen was chiefly obtained from the caliche deposits in Chile, and the rate of extraction was so fast that in 1898 Crookes predicted a world famine in nitrogen, and therefore in agricultural products, at no very distant date. Within a very few years, the situation was changed by the establishment of the first plant for the production of nitric acid from atmospheric nitrogen. That first process, depending on the electric arc, has now been superseded, first by the cyanamide process, and then by the Haber process, in which the immediat ${ }^{3}$ product is ammonia, instead of oxides of nitrogen. It is of interest to note that the arc process, with the production of oxides, is the one used by Nature herself as the main agency for restoring nitrogen to the earth's surface for agricultural needs; her arc is the lightning flash. In 1937 there were 145 plants operating on the Haber process, producing nearly 4 million tons of fixed nitrogen, an amount which, though large in itself, is only the amount to be found above 1/5 square mile of the earth's surface. In any event, the nitrogen withdrawn from the air almost all ultimately finds its way back again, through the agency of living matter.

From the consideration of these matters, we are eventually reminded that the balance of Nature is the only basis of man's existence. It will demand control over vegetation which has been lacking ever since the inception of the industrial period, and it is to chemists that the world should look for a lead.

\section{Factor Analysis}

$A^{T}$

$T$ the general meeting of the British Psychological Society, held this year at the University of Reading, a symposium on factor analysis attracted on April 1 the largest number of members present for several years at any meeting of the Society.

Opening the symposium, Prof. Godfrey Thomson said that many sets of factors are mathematically available, and principles have to be adopted for deciding between them. These have included: (1) the universal desire to use few common factors ; (2) reproducing the whole variance; (3) reproducing the correlations ; (4) insistence on a general factor ; (5) rotating to psychological significance; (6) requiring 'simple structure'; (7) requiring invariance of analysis; (8) requiring reciprocity of persons and tests. The second loses least information, but conflicts with (5) and (7). The third principle is used by both the Spearman and Thurstone schools; the former proceeds step by step, the latter by a simultaneous analysis. The Spearman school safeguards psychological significance at each step, and insists on a general factor. The Thurstone plan rotates the factors into 'simple structure' and holds that psychological significance will ensue. 'Simple structure' seems to rule out a general factor, and Thurstone finds no $g$ in Spearman's own $g$-tests. Both methods result in large specific factors, and have more factors than tests, so that the factors cannot be accurately estimated. The last principle (8) is put forward by Burt, who analyses covariances. His reciprocity is only obtained, however, after removal of a factor composed of the average.

Prof. Spearman's paper, read by Mr. Maxwell Garnett, directed attention to the disappearance of the general factor in Thurstone's "Primary Mental Abilities". A set of correlations, he said, can be factorized in many ways, the choice being regulated by statistical simplicity and psychological interest. The theory of two factors uses a method which draws all the correlations from one factor. Statistically simple, it affords psychologically the basis for the concepts of general ability. Hotelling, Kelley and Burt, in securing that the weights of factors are heaped up in order of calculation, generate negative loadings which contradict experience. Alexander and Thurstone, in rejecting negative loadings, decrease without eliminating the indeterminateness. Thurstone in addition maximizes the number of zeros. It is this which banishes the general factor, for weights which fall below a certain minimum are ascribed to error and counted as zero. Moreover the number of Thurstone's factors creates suspicion.

Correlations alone fail to determine factors completely: other grounds are needed. In the special case of one single factor we have the cogent ground of parsimony; in all other cases to find adequate grounds is difficult.

Prof. Burt emphasized that the components obtained by factorizing a correlation table describe an analysis of the relations between minds and their environments. Whether a factor has meaning depends on the appropriateness of the experimental design as much as on the validity of the analysis. Factor analysis should, if possible, be based on covariances rather than on correlations. Variance varies objectively, and depends primarily on the complexity of the process measured. When the errors are sufficiently small, all analyses reveal a series of factors whose contributory variances are roughly $50,12,5$ . . per cent of the total. This may be explained by regarding factor analysis as a process of successive averaging. Concerning the 'sampling theory', he said that an effective test integrates a wide variety rather than samples a large number of co-ordinations. 